\title{
SÍNDROME PULMONAR POR HANTAVIRUS (VIRUS RÍO MAMORÉ) EN LA AMAZONÍA PERUANA
}

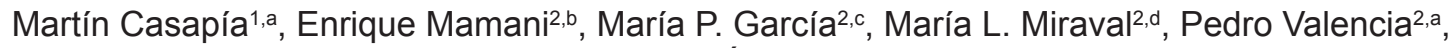 \\ Alberto H. Quino ${ }^{1, e}$, Carlos Álvarez ${ }^{3, d}$, Luis F. Donaires ${ }^{4, f}$
}

\begin{abstract}
RESUMEN
La hantavirosis es una infección viral zoonótica transmitida por roedores cuya forma clínica más letal es el síndrome pulmonar por Hantavirus (SPH). La variante río Mamoré es autóctona de Sudamérica y fue descrita en roedores sin asociarla a enfermedad en humanos. Se presenta dos casos de SPH causados por hantavirus río Mamoré en la Amazonía peruana en noviembre de 2011. En ambos casos, el diagnóstico confirmatorio fue molecular, efectuados en el Instituto Nacional de Salud de Perú. Se realizó análisis filogenético del fragmento de genoma viral y la evaluación histopatológica. Ambos pacientes evolucionaron a síndrome de distrés respiratorio del adulto y estado de choque refractario. Un paciente falleció y el otro se recuperó a los doce días.
\end{abstract}

Palabras clave: Hantavirus; Síndrome pulmonar por hantavirus; Perú (fuente: DeCS BIREME).

\section{HANTAVIRUS PULMONARY SYNDROME (RIO MAMORE VIRUS) IN THE PERUVIAN AMAZON REGION}

\begin{abstract}
Hantavirus infection is a viral zoonotic infection borne by rodents which most letal form clinical is the Hantavirus Pulmonary Syndrome (SPH, Spanish abbreviation). The Mamore River variant originates in South America and was found in rodents without any association to human diseases. Two cases of SPH were identified in the Peruvian Amazon region in November 2011. In both cases, a molecular diagnostic testing was conducted by the Instituto Nacional de Salud from Peru. A phylogenetic analysis of a viral genome fragment and a histopathological evaluation were conducted. Both patients developed adult respiratory distress syndrome and refractory shock. A patient died and another one recovered 12 days later.
\end{abstract}

Key words: Hantavirus; Hantavirus pulmonary syndrome; Peru (source: MeSH NLM).

\section{INTRODUCCIÓN}

La hantavirosis es una zoonosis viral trasmitida por roedores. La enfermedad es causada por un virus del género Hantavirus, familia Bunyaviridae, que se transmite principalmente a los humanos por la inhalación de aerosoles generados por la orina y las heces de roedores infectados ${ }^{(1)}$.

El primer brote de síndrome pulmonar por Hantavirus $(\mathrm{SPH})$ en el continente americano ocurrió en los EE. UU. en 1993, identificándose como agente etiológico el Hantavirus, denominado virus sin nombre (SNV) ${ }^{(2)}$. En Sudamérica, el primer Hantavirus asociado a SPH fue descrito en Brasil (1993) ${ }^{(3)}$ y el primer reporte realizado en Bolivia (1996) le denominó Hantavirus río Mamoré (RIOMV) ${ }^{(4)}$. Su caracterización genética fue realizada un año después por Bharadwaj M. et al. estudiando roedores silvestres (Oligoryzomys microtis) procedentes de los departamentos de La Paz y Beni en Bolivia ${ }^{(5)}$. En lquitos, ciudad ubicada en el noreste peruano, Powers A. et al. (1996) identificaron una variante del RIOMV en O. microtis ${ }^{(6)}$.

\footnotetext{
Hospital Regional de Loreto, Gobierno Regional de Loreto. Loreto, Perú.

Centro Nacional de Salud Pública. Instituto Nacional de Salud. Lima, Perú.

Dirección Regional de Salud de Loreto, Gobierno Regional de Loreto. Loreto, Perú.

Unidad de Análisis y Generación de Evidencias en Salud Pública. Instituto Nacional de Salud. Lima, Perú.

Médico infectólogo; ${ }^{\mathrm{b}}$ biólogo; ${ }^{\mathrm{c}}$ tecnólogo médico ${ }^{\mathrm{d}}$ médico anátomo-patólogo; ${ }^{\mathrm{e}}$ médico intensivista; ${ }^{\mathrm{f}}$ médico epidemiólogo

Recibido: 09-02-12 Aprobado: 26-06-12
}

Citar como: Casapía M, Mamani E, García MP, Miraval ML, Valencia P, Quino AH, et al. Síndrome pulmonar por hantavirus (virus Río Mamoré) en la Amazonía peruana. Rev Peru Med Exp Salud Publica. 2012;29(3):390-5. 
Richter M. et al. estudiaron la relación filogenética entre el hantavirus identificado en el Perú con otros hantavirus, y encontró que el análisis de nucleótidos y la secuencia de aminoácidos correspondía a una variante del RIOMV el cual es enzoótico en O. microtis, su huésped principal ${ }^{(7)}$.

Aunque el RIOMV hasta ahora no se había asociado con enfermedad en humanos, Carroll et al. reportaron el RIOMV en un O. microtis en la región Mineros de Bolivia, donde había fallecido una persona por SPH; sin embargo, no se tomó muestra del caso fatal para compararla con la secuencia viral del roedor. Por la proximidad geográfica de la captura del roedor con PCR positivo para RIOMV y el lugar donde el paciente se enfermó, parecería posible que esta variante de RIOMV pueda ser el patógeno responsable del caso de $\mathrm{SPH}{ }^{(8)}$

En 1998, un estudio de seroprevalencia de anticuerpos IgG, utilizando ELISA para hantavirus en muestras colectadas de escolares (1993 a 1996), pacientes febriles (1995 a 1996) y roedores (1997) de la ciudad de lquitos, encontró una prevalencia de $2 \%$ (30/1537); $8,2 \%$ $(21 / 257)$, y $3,5 \%$ (4/112) respectivamente, reactivos para el virus Hantaan y SNV ${ }^{(9)}$. Adicionalmente, Castillo et al. en una investigación de pacientes febriles en lquitos encontró una prevalencia de $0,3 \%$ (15/5174) con IgM reactivo para Hantavirus (virus Andes), todos con enfermedad leve y autolimitada ${ }^{(10)}$. En julio de 2011, se informaron en la ciudad de lquitos los dos primeros casos de hantavirosis humana en el Perú, causados por el virus Seoul (SEOV) ${ }^{(11)}$.

En este reporte describimos dos casos de SPH (noviembre 2011) causados por el virus RIOMV procedentes de dos comunidades rurales de la Amazonía peruana, separadas por una distancia de $5 \mathrm{~km}$. Todas las pruebas de confirmación diagnóstica fueron realizadas en el Instituto Nacional de Salud de Perú.

\section{REPORTE DE CASO}

Las características epidemiológicas, clínicas y de laboratorio se resumen en las tablas 1 y 2 .

\section{CASO 1}

Siete días antes del ingreso hospitalario, inició enfermedad con sensación de alza térmica no cuantificada, dolor abdominal en hemicuadrante superior, deposiciones líquidas sin moco ni sangre en múltiples oportunidades y tos seca que gradualmente se hizo intensa. Dos días antes del ingreso se
Tabla 1. Características epidemiológicas y diagnósticas de dos casos de síndrome pulmonar por Hantavirus producido por el virus RIOMV en la Amazonía peruana, 2011.

\begin{tabular}{|c|c|c|}
\hline & Caso 1 & Caso 2 \\
\hline Edad, sexo & $18, M$ & $38, F$ \\
\hline $\begin{array}{l}\text { Localidad de procedencia } \\
\text { (centro poblado/distrito) }\end{array}$ & $\begin{array}{l}\text { Timicurillo/ } \\
\text { Indiana }\end{array}$ & $\begin{array}{l}\text { Nuevo Varade- } \\
\text { rillo/ } \\
\text { Mazán }\end{array}$ \\
\hline Zona de residencia & Rural & Rural \\
\hline $\begin{array}{l}\text { Distancia al primer ais- } \\
\text { lamiento RIOMV en un } \\
\text { roedor (año 1996) }{ }^{(6)}\end{array}$ & $75 \mathrm{~km}$ & $80 \mathrm{~km}$ \\
\hline Ocupación & Agricultor & $\begin{array}{l}\text { Agricultora/ } \\
\text { ama de casa }\end{array}$ \\
\hline Diagnóstico inicial & $\begin{array}{c}\text { Shock } \\
\text { séptico, } \\
\text { leptospirosis } \\
\text { pulmonar, } \\
\text { Hantavirus }\end{array}$ & $\begin{array}{l}\text { NAC, leptospi- } \\
\text { rosis pulmonar, } \\
\text { Hantavirus }\end{array}$ \\
\hline \multicolumn{3}{|l|}{ Método diagnóstico } \\
\hline ELISA IgM & $1 / 6400$ & $1 / 1600$ \\
\hline $\begin{array}{l}\text { Inmunofluorescencia } \\
\text { indirecta }\end{array}$ & $1 / 32$ & $1 / 16$ \\
\hline RT-PCR nested en suero & Positivo & Positivo \\
\hline $\begin{array}{l}\text { Secuenciación genética } \\
\text { (Fragmento } 210 \mathrm{pb} \\
\text { segmento S) }\end{array}$ & RIOMV & RIOMV \\
\hline Cultivo viral & Negativo & Negativo \\
\hline Clasificación & Confirmado & Confirmado \\
\hline
\end{tabular}

RT-PCR: transcripción reversa - reacción en cadena de la polimerasa; ELISA: Enzyme-Linked Immuno Sorbent Assay (ensayo por inmunoabsorción ligado a enzimas); IgM: inmunoglobulina M; NAC:neumonía adquirida en la comunidad

instauró disnea progresiva con retracción intercostal. Paciente fue admitido en mal estado general; pálido; polipneico; soporoso con cianosis peribucal; presión arterial 100/64 mmHg; pulso: 95 lat/min; frecuencia respiratoria: $38 \mathrm{resp} / \mathrm{min}$; temperatura: $37,5{ }^{\circ} \mathrm{C}$; $\mathrm{SatO}_{2} 92 \%\left(\mathrm{FiO}_{2}:\right.$ 0,5); presentando un episodio de vómito porráceo. En el examen de tórax se evidenció retracciones intercostales, presencia de crepitantes en ambos hemitórax y hepatoesplenomegalia. Durante la intubación para soporte ventilatorio mecánico se obtiene aspirado bronquial hemático. Los resultados de los exámenes auxiliares mostraron leucocitosis sin desviación izquierda, retención nitrogenada y signos de insuficiencia respiratoria hipoxémica en la gasometría arterial (Tabla 2). La radiografía de tórax de admisión mostró infiltrado alveolar multilobar bilateral e infiltrado alveolointersticial apical izquierdo (Figura 1A). 

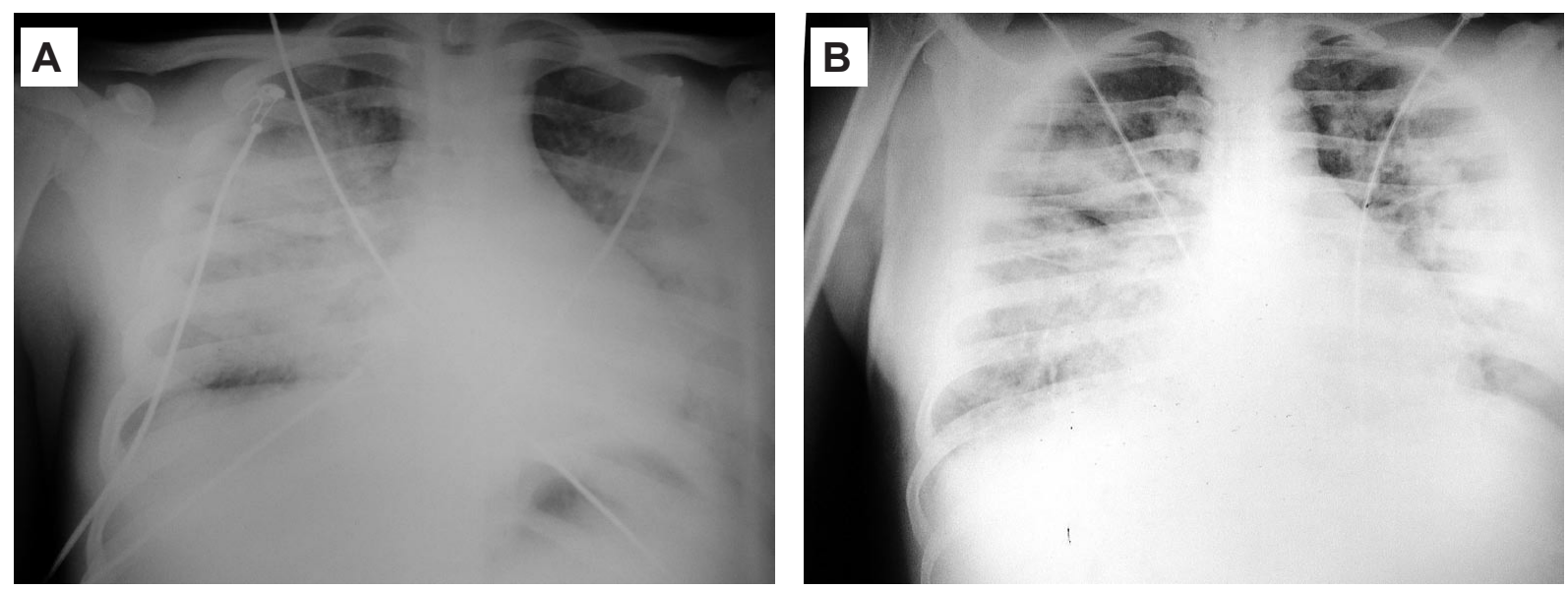

Figura 1. Radiografía de tórax al ingreso. (A) Caso 1: infiltrado alveolar multilobar bilateral e infiltrado alveolointersticial apical izquierdo. (B) Caso 2: infiltrado alveolar multilobar bilateral confluente e infiltrado alveolo nodular apical izquierdo.

Tabla 2. Datos de laboratorio de dos casos de síndrome pulmonar por Hantavirus producido por el virus RIOMV en la Amazonía peruana, 2011.

\begin{tabular}{|c|c|c|}
\hline & Caso 1 & Caso 2 \\
\hline \multicolumn{3}{|l|}{ Hemograma inicial } \\
\hline Hematocrito & $42 \%$ & $55 \%$ \\
\hline Recuento de plaquetas & $156000 / \mathrm{mm}^{3}$ & $145000 / \mathrm{mm}^{3}$ \\
\hline Leucocitos & $15700 \mathrm{cél} / \mathrm{mm}^{3}$ & $14000 \mathrm{cél} / \mathrm{mm}^{3}$ \\
\hline Segmentados & $79 \%$ & $84 \%$ \\
\hline Abastonados & $1 \%$ & - \\
\hline Linfocitos & $18 \%$ & $15 \%$ \\
\hline \multicolumn{3}{|c|}{ Bioquímica sanguínea (mg/dL) } \\
\hline Glucosa & 167,7 & 148,0 \\
\hline Creatinina & 1,6 & 3,4 \\
\hline Urea & 67,5 & 88,0 \\
\hline \multicolumn{3}{|c|}{ Gasometría arterial (mmHg) } \\
\hline $\mathrm{pH}$ & 7,2 & 7,4 \\
\hline $\mathrm{PO}_{2}$ & 77,1 & 57,3 \\
\hline $\mathrm{PCO}_{2}$ & 20,0 & 23,1 \\
\hline $\mathrm{HCO}_{3}^{-}$ & $7,8 \mathrm{mmol} / \mathrm{L}$ & $13,0 \mathrm{mmol} / \mathrm{L}$ \\
\hline $\mathrm{FiO}_{2}$ & 0,5 & 0,4 \\
\hline $\mathrm{Pa} / \mathrm{FiO}_{2}$ & 154,0 & 143,3 \\
\hline $\mathrm{Na}^{+}$ & $126,2 \mathrm{mmol} / \mathrm{L}$ & $125,0 \mathrm{mmol} / \mathrm{L}$ \\
\hline $\mathrm{K}^{+}$ & $3,5 \mathrm{mmol} / \mathrm{L}$ & $4,3 \mathrm{mmol} / \mathrm{L}$ \\
\hline
\end{tabular}

A las doce horas de admisión, el paciente presentó estado de choque, se inició soporte inotrópico con dopamina y dobutamina sin respuesta, agregándosele adrenalina en infusión. Asimismo, presentó hemoneumotórax derecho, posterior a la colocación de catéter venoso central, seguido de sangrado profuso posdrenaje torácico que requirió de trasfusión sanguínea. A las 48 horas, presentó convulsiones focalizadas en miembro superior izquierdo y anisocoria. La tomografía cerebral mostró sangrado intraparenquimal en ambos hemisferios cerebrales y hemorragia subaracnoidea. A las 72 horas, presentó insuficiencia renal aguda. A los cinco días de hospitalización se recibe la confirmación serológica (ELISA IgM: 1/6400 e IFI IgM: 1/32) y molecular de Hantavirus. EI estudio laboratorial de hepatitis viral, leptospirosis, dengue, influenza A, parainfluenza, adenovirus, metaneumovirus y virus sincitial respiratorio fueron negativos. Falleció a los seis días de hospitalización en UCl por falla multiorgánica.

El estudio histopatológico de tejido hepático mostró amplias áreas de necrosis en parches, principalmente en la zona I y II del lobulillo con congestión sinusoidal y focos de hemorragia; asimismo, se observaron cuerpos de Councilman e infiltrado inflamatorio linfocitario distribuido dentro del parénquima (Figura 2A). En los pulmones se encontró signos de neumonitis descamativa con focos de edema, congestión vascular severa y presencia de trombos hemáticos (Figura 2B). En el tejido renal se apreció intensa degeneración hidrópica de células tubulares, congestión y trombosis vascular con leve infiltrado linfocitario a nivel intersticial, los túbulos colectores estuvieron preservados (Figura 2C). Asimismo, se encontró edema e infiltrado inflamatorio linfocitario intersticial en parénquima cerebral, bazo, páncreas y músculo cardiaco.

\section{CASO 2}

Cinco días antes de ingreso hospitalario, inició enfermedad con dolor osteomuscular, sensación de alza térmica, dolor epigástrico y náuseas. Luego de dos días presentó vómitos con deposiciones líquidas sin moco ni sangre, y $24 \mathrm{~h}$ después se agregaron tos productiva con expectoración verdosa y disnea que gradualmente se intensificó. Fue admitida en mal estado general con presión arterial: $95 / 67 \mathrm{mmHg}$; frecuencia cardiaca: 100 lat $/ \mathrm{min}$; frecuencia respiratoria: $44 \mathrm{resp} / \mathrm{min}$; temperatura: $36,8^{\circ} \mathrm{C}$, y 

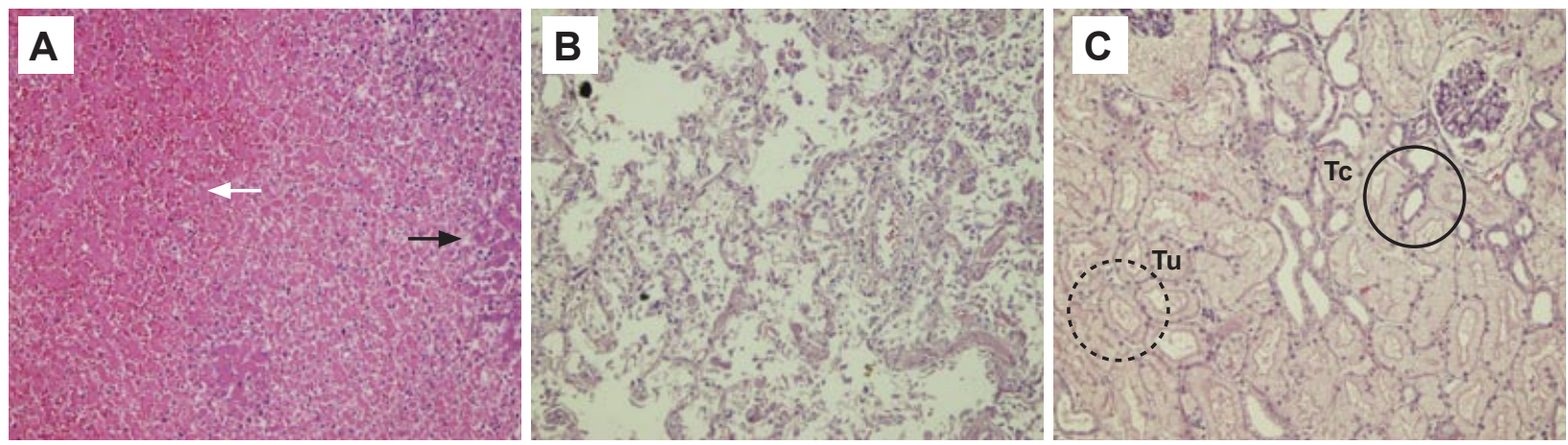

Figura 2. Muestras histológicas. (A) Tejido hepático con amplias áreas de necrosis (flecha blanca), congestión sinusoidal y focos hemorrágicos (flecha negra). (B) Tejido pulmonar con signos de neumonitis descamativa. (C) Tejido renal con intensa degeneración hidrópica de tubulis (Tu) y preservación de túbulos colectores(Tc) (coloración HE aumento 200X).

SatO $_{2} 87,5 \%\left(\mathrm{FiO}_{2}: 0,21\right)$. El examen de tórax evidenció retracciones intercostales y subcostales, crepitantes y subcrepitantes en ambos hemitorax y hepatomegalia. Los resultados de los exámenes auxiliares mostraron leucocitosis sin desviación izquierda, retención nitrogenada y signos de insuficiencia respiratoria hipoxémica en la gasometría arterial (Tabla 2). La radiografía de tórax al ingreso evidenció infiltrado alveolar multilobar bilateral confluente a predominio de dos tercios inferiores e infiltrado alveolonodular apical izquierdo (Figura 1B). Ingresó a $\mathrm{UCl}$ con criterios de síndrome de distrés respiratorio agudo del adulto (SDRA), iniciándose ventilación mecánica; desarrolló estado de choque que no respondió a dopamina ni dobutamina por lo que se le inició adrenalina con respuesta favorable. Asimismo, presentó insuficiencia renal aguda que requirió hemodiálisis. A los tres días se recibe la confirmación serológica (ELISA IgM: 1/1600 e IFI IgM: 1/16) y molecular de Hantavirus. El rt-PCR de hisopado nasofaríngeo fue negativo a Influenza A y B. La paciente evolucionó de manera favorable siendo dada de alta a los 12 días.

\section{ANÁLISIS FILOGENÉTICO}

El análisis filogenético del fragmento de $210 \mathrm{pb}$, del segmento genómico $S$ de ambos casos, presentó alta homología (96\%) con la secuencia del virus Rio Mamoré, aislado en un roedor de la especie Oligoryzomys microtis en Iquitos el año 1996 (Figura 3).

\section{DISCUSIÓN}

Se describen dos casos de hantavirosis producido por el RIOMV, con manifestaciones clínicas compatibles con SPH; los casos se presentaron en una región donde previamente se reportó la circulación del RIOMV en roedores silvestres ${ }^{(6)}$.
La alta homología del secuenciamiento del fragmento de genoma viral amplificado encontrada con el RIOMV, el antecedente de su aislamiento previo en un roedor silvestre (Oligorizomys microti) y la procedencia rural de los dos casos reportados, hacen suponer que este virus tiene una amplia distribución en la región amazónica (7).

La presentación clínica inicial de los casos estuvieron caracterizados por un cuadro febril, malestar general, dolor abdominal, diarrea acuosa, tos, cianosis, insuficiencia respiratoria aguda grave e infiltrado alveolar o intersticial bilateral consistente con SDRA; características clínicas concordantes con las descritas en los 510 casos de SPH confirmados por el CDC en los EE. UU. (12). Otros hallazgos característicos del SPH fueron la presencia de leucocitosis sin desviación izquierda, la disminución leve del número plaquetas, así como la elevación del hematocrito, la creatinina y la urea. Asimismo, se encontró hipoxemia refractaria con $\mathrm{Pa} / \mathrm{FiO}_{2}$ menores de 200, indicación de ventilación mecánica.

La evolución de los casos fue tórpida, llegaron a disfunción multiorgánica con SDRA, insuficiencia renal y estado de choque refractario en ambos casos, y signos de eventos hemorrágicos múltiples en el caso fatal; estos hallazgos fueron similares a los reportados en los brotes de SPH presentados en los EE. UU. y países sudamericanos ${ }^{(13)}$.

Los hallazgos histopatológicos del caso fatal, mostraron signos de edema e infiltrado linfocitario en la totalidad de órganos evaluados. Se evidenció necrosis extensa del parénquima hepático con presencia de amplias áreas de sangrado, lo que sugeriría un papel hepatotropo del virus RIOMV. Reportes de estudios histopatológicos realizados en tejido pulmonar del SPH muestran conservación de la arquitectura alveolar con 


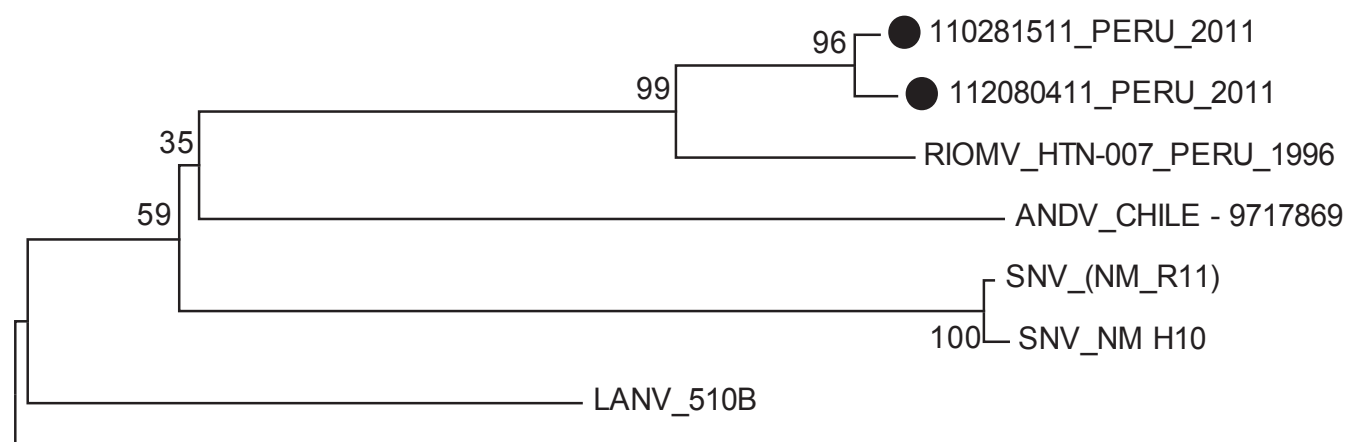

PUUV/Mg23/HungaryTR17/00

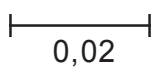

Figura 3. Árbol filogenético de un fragmento de 210pb, del segmento genómico S, de dos casos de SPH (Caso 1: 110281511, Caso 2: 112080411), utilizando el método de análisis filogenético Maximum Likelihood con 500 repeticiones.

RIOMV: virus río Mamoré, ANDV: virus Andes, SNV: virus sin nombre, LANV: virus Laguna Negra, PUUV: virus Pumala.

signos de neumonitis intersticial y presencia de inmunoblastos ${ }^{(14)}$, hallazgos no observados en el caso descrito que presentó signos de neumonitis descamativa con focos de edema y severa congestión vascular. La degeneración hidrópica intensa de las células tubulares encontradas en el caso fatal, también fueron reportados en infecciones experimentales de macacos con Hantavirus Puumala ${ }^{(15)}$ causante de la fiebre hemorrágica con síndrome renal y en autopsias de casos de SPH por virus ANDV (14).

En razón que no se dispone de tratamiento específico para $\mathrm{SPH}$, en la actualidad el único tratamiento continúa siendo el de soporte. Ambos casos, fueron manejados en unidades críticas, donde se le dio soporte ventilatorio y hemodinámico con sustancias vasopresoras e inotrópicas. La clave del tratamiento sigue siendo el reconocimiento temprano de la enfermedad, lo cual es un reto para el clínico, sobre todo en las fases prodrómicas ${ }^{(13)}$.

En conclusión, se confirmaron dos casos de SPH producidos por el RIOMV, cuyas manifestaciones clínicas iniciales fueron muy inespecíficas, pero una vez establecida la enfermedad, los compromisos respiratorio y cardiovascular fueron rápidamente progresivos, conllevando al deceso de uno de los casos.

Agradecimientos: al Instituto Nacional de Enfermedades Virales Humanas, Pergamino de Argentina, por facilitarnos las pruebas de ELISA IgM con antígeno inactivado del virus Maciel. Al Dr. Antonio Tenorio del Instituto Carlos III, Madrid, España, por proporcionarnos el protocolo de la transcripción reversa reacción en cadena de la polimerasa y análisis de secuenciación. Al personal profesional del INS, Susy Merino, Dina Torres, Dana Figueroa, Marco Coaguila, Noelia Milla del Laboratorio de Referencia Nacional de Metaxénicas Virales; al técnico de laboratorio Raúl Román del Laboratorio de Patología, por su labor en la investigación laboratorial de los casos presentados.

Contribuciones de autoría: MC, EM,LFD, MPG, MLM, HAQ y CA participaron en la concepción del estudio. MC, EM,LFD, MPG y MLM en el diseño; MC, HAQ y CA en el aporte de pacientes y material de estudio; MC, EM,LFD, MPG, MLM y HAQ en el análisis de resultados; MC, LFD y PV en la revisión crítica del manuscrito, PV en la obtención del financiamiento; MC y EM en la aprobación de la versión final.

Fuentes de financiamiento: Instituto Nacional de Salud de Perú y Hospital Regional de Loreto.

Conflictos de interés: los autores declaran no tener conflictos de interés en la publicación de este artículo. 


\section{REFERENCIAS BIBLIOGRÁFICAS}

1. Hart CA, Bennett M. Hantavirus infections: epidemiology and pathogenesis. Microbes Infect. 1999;1(14):1229-37.

2. Nichol ST, Spiropoulou CF, Morzunov S, Rollin PE, Ksiazek TG, Feldmann $\mathrm{H}$, et al. Genetic identification of a hantavirus associated with an outbreak of acute respiratory illness. Science. 1993;262(5135):914-7.

3. Johnson AM, de Souza LT, Ferreira IB, Pereira LE, Ksiazek TG, Rollin PE, et al. Genetic investigation of novel hantaviruses causing fatal HPS in Brazil. J Med Virol. 1999;59(4):527-35.

4. Hjelle B, Torrez-Martinez N, Koster FT. Hantavirus pulmonary syndrome-related from Bolivia. Lancet. 1996;347(8993):57.

5. Bharadwaj M, Botten J, Torrez-Martinez N, Hjelle B. Rio Mamore Virus: Genetic characterization of a newly recognized Hantavirus of the pygmy rice rat, Oligoryzomys microtis, from Bolivia. Am J Trop Med Hyg. 1997;57(3):36874.

6. Powers AM, Mercer DR, Watts DM, Guzman H, Fulhorst CF, Popov VL, et al. Isolation and genetic characterization of a hantavirus (Bunyaviridae: Hantavirus) from a rodent, Oligoryzomys microtis (Muridae), collected in northeastern Peru. Am J Trop Med Hyg. 1999;61(1):92-8.

7. Richter M, Hanson JD, Cajimat N, MilazzoML, Fulhorst Ch. Geographical range of Rio Mamoré virus (family Bunyaviridae, genus Hantavirus) in association with the small-eared pygmy rice rat (Oligoryzomys microtis). Vector Borne Zoonotic Dis. 2010;10(6):613-20.

8. Carroll DS, Mills JN, Montgomery JM, Bausch DG, Blair PJ, Burans JP, et al. Hantavirus pulmonary syndrome in Central Bolivia: relationships between reservoir hosts, habitats, and viral genotypes. Am J Trop Med Hyg. 2005;72(1):42-6.

9. Cilloniz C. Evidencia serológica del virus Hantaan, Sin Nombre y Puumala en la región amazónica de Iquitos [Tesis para optar el grado de Magister en Microbiología]. Lima, Perú: Facultad de Medicina, UNMSM; 1998.

10. Castillo Oré RM, Forshey BM, Huaman A, Villaran MV, Long KC, Kochel TJ, et al. Serologic evidence for human Hantavirus infection in Peru. Vector Borne Zoonotic Dis. 2012 May 22. [Epub ahead of print].

11. García MP, Percy S, Herrera AL, Donaires F, Alvarez C, Arrasco J, et al. Etiologic confirmation of the first two cases of hu- man hantavirosis in Perú. Rev Peru Med Exp Salud Publica. 2011;28(3):566-7.

12. MacNeil A, Ksiazek TG, Rollin PE. Hantavirus pulmonary syndrome, United States, 1993-2009. Emerg Infect Dis. 2011;17(7):1195-201.

13. Chile, Ministerio de Salud. Diagnóstico y manejo del síndrome cardiopulmonar por hantavirus. Guía Clínica. 2da Ed. Santiago, Chile: MISAL; 2009.

14. Guzmán P, Tapia O, Villaseca M, Araya J, Antonio L, Lee B, et al. Hallazgos morfológicos en casos fatales de síndrome cardiopulmonar por hantavirus. Estudio de 7 autopsias. Rev Chil Infectol. 2010;27(5):398-405.

15. Sironen T, Klingström J, Vaheri A, Andersson LC, Lundkvist A, Plyusnin A. Pathology of Puumala hantavirus infection in macaques. PLoS One. 2008;3(8):e3035.

Correspondencia: Martin Casapia

Dirección: Urb. Jardin 27 (Fanning 4. ${ }^{\text {ta }}$ cuadra), Iquitos, Perú.

Teléfono: (51) 965621830

Correoelectrónico:mcasapia@acsaperu.org

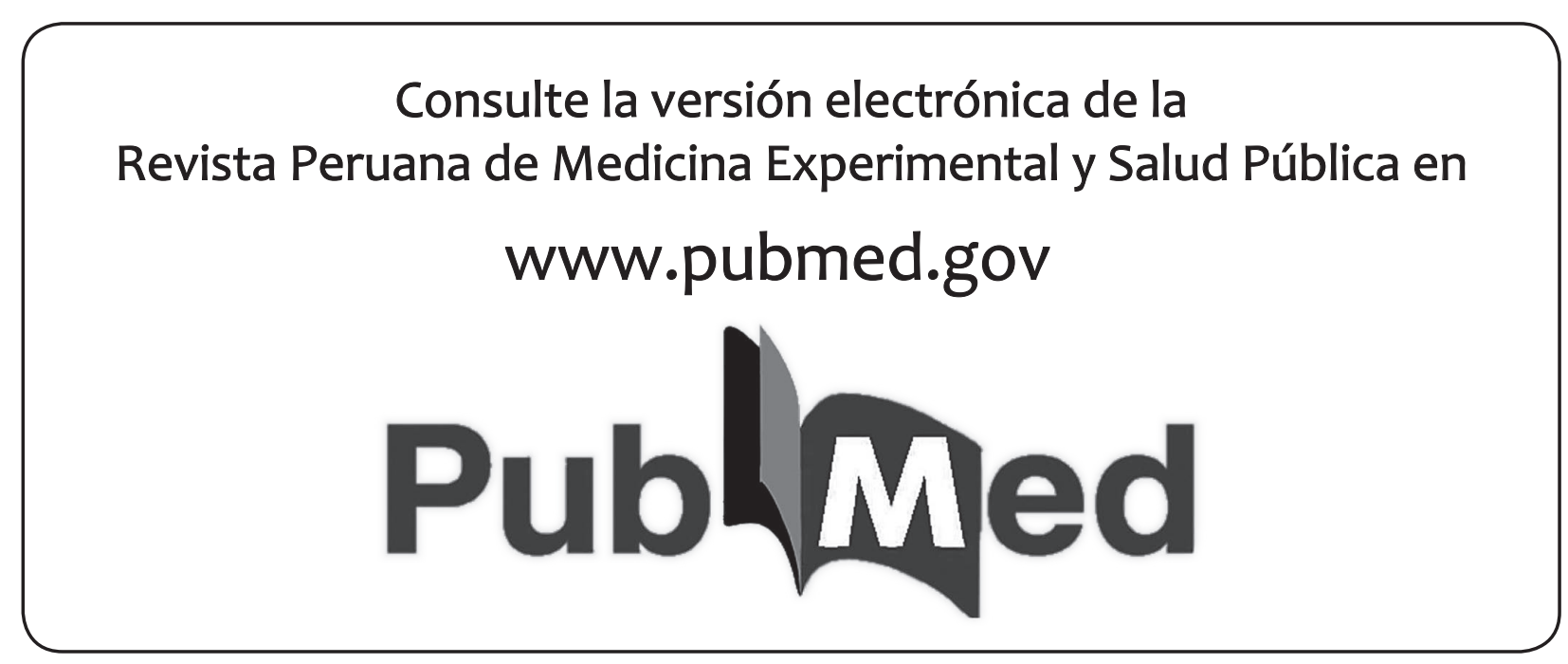

